\title{
Surgeon interrater reliability in the endoscopic assessment of cistern scarring and aqueduct patency
}

\author{
Lucy He, MD, ${ }^{1}$ Stephen Gannon, BS, ${ }^{1}$ Chevis N. Shannon, MPH, MBA, DrPH, ${ }^{1}$ \\ Brandon G. Rocque, MD, MS, ${ }^{2}$ Jay Riva-Cambrin, MD, MSc, ${ }^{3}$ and Robert P. Naftel, MD1 \\ 'Department of Neurological Surgery, Vanderbilt University Medical Center, Nashville, Tennessee; ${ }^{2}$ Pediatric Neurosurgery, \\ Department of Neurosurgery, University of Alabama at Birmingham, Alabama; and 'Division of Pediatric Neurosurgery, \\ Department of Clinical Neurosciences, Alberta Children's Hospital, Calgary, Alberta, Canada
}

\begin{abstract}
OBJECTIVE The success of endoscopic third ventriculostomy with choroid plexus cauterization may have associations with age, etiology of hydrocephalus, previous shunting, cisternal scarring, and possibly aqueduct patency. This study aimed to measure interrater reliability among surgeons in identifying cisternal scarring and aqueduct patency.

METHODS Using published definitions of cistern scarring and aqueduct patency, 7 neuroendoscopists with training from Dr. Warf in Uganda and 7 neuroendoscopists who were not trained by Dr. Warf rated cistern status from 30 operative videos and aqueduct patency from 26 operative videos. Interrater agreement was calculated using Fleiss' kappa coefficient (к). Fisher's 2-tailed exact test was used to identify differences in the rates of agreement between the Warftrained and nontrained groups compared with Dr. Warf's reference answer.
\end{abstract}

RESULTS Aqueduct status, among all raters, showed substantial agreement with $\kappa=0.663$ (confidence interval [Cl] $0.626-0.701)$; within the trained group and nontrained groups, there was substantial agreement with $\kappa=0.677$ (Cl $0.593-0.761)$ and $\kappa=0.631$ (Cl 0.547-0.715), respectively. The identification of cistern scarring was less reliable, with moderate agreement among all raters with $\kappa=0.536(\mathrm{Cl} 0.501-0.571)$; within the trained group and nontrained groups, there was moderate agreement with $\kappa=0.555(\mathrm{Cl} 0.477-0.633)$ and $\kappa=0.542(\mathrm{Cl} 0.464-0.620)$, respectively. There was no statistically significant difference in the amount of agreement between groups compared with Dr. Warf's reference.

CONCLUSIONS Regardless of training with Dr. Warf, all neuroendoscopists could identify scarred cisterns and aqueduct patency with similar reliability, emphasizing the strength of the published definitions. This makes the identification of this risk factor for failure generalizable for surgical decision making and research studies.

http://thejns.org/doi/abs/10.3171/2016.3.PEDS15648

KEY WORDS endoscopic third ventriculostomy; choroid plexus cauterization; hydrocephalus; risk factors

$\mathrm{H}$ YDROCEPHALUS is a disorder of CSF circulation that causes increased intracranial pressure. Traditional treatment for this condition involves CSF diversion with a shunt that diverts CSF from the head to a distal absorptive space (e.g., peritoneum, pleura, atria). Unfortunately, shunts carry a risk of infection and shunt failure, often requiring multiple revisions over a patient's lifetime. Studies have found that up to $84.5 \%$ of patients require at least 1 shunt replacement and $4.7 \%$ require 10 or more over the course of 17 years. ${ }^{7}$ An ideal hydrocephalus procedure provides long-term control while minimizing surgical interventions.

Endoscopic third ventriculostomy (ETV) with choroid plexus cauterization (CPC) is an evolving therapy for hydrocephalus in young children and an alternative to shunting. ${ }^{7,11}$ Factors associated with success have consistently been age, etiology of hydrocephalus, previous shunting, and cisternal scarring; aqueduct patency may also be related to success. ${ }^{11,12}$ Age, etiology, and previous shunting are objectively defined. Cisternal scarring and aqueduct patency are subjectively defined and require surgeons to visually rate the cistern as clean or scarred and the aqueduct as open or closed. In Warf et al.'s studies, these endoscopic findings were specifically defined. ${ }^{11,12}$ For the purposes of training new surgeons to perform ETV/CPC and researching predictive factors, the reliability of surgeons

ABBREVIATIONS CPC = choroid plexus cauterization; ETV = endoscopic third ventriculostomy.

SUBMITTED November 4, 2015. ACCEPTED March 21, 2016.

INCLUDE WHEN CITING Published online May 27, 2016; DOI: 10.3171/2016.3.PEDS15648. 
to identify these findings must be defined. Additionally, the generalizability of these definitions was studied by comparing the ability of surgeons who trained with Warf to surgeons who perform endoscopy but have not trained with Dr. Warf. This study tested the interrater reliability of these 2 groups of surgeons in identifying cisternal scarring and aqueductal patency using Warf's definitions.

\section{Methods}

\section{Patients}

Prior to the selection of the patient videos, institutional review board approval was obtained. A series of videos of ETV/CPC were collected from the procedures performed at CURE Children's Hospital of Uganda, Vanderbilt Children's Hospital, Children's of Alabama, and Primary Children's Hospital. Cases were edited to capture characteristic videos of the prepontine cistern and cerebral aqueduct. No identifiable patient information was collected, and there were no identifiable features in the videos that could have led to the identification of the patients.

A total of 30 cistern videos and 26 aqueduct videos were chosen. Each video clip was approximately $10 \mathrm{sec}-$ onds long. For the cistern videos, the clip was edited to include views of the fenestrated third ventricle floor and then a view "through" the ventriculostomy showing the basilar cistern space. For the aqueduct videos, a reflexed view with a flexible scope was undertaken to allow a magnified, unobstructed view of the aqueduct ostium. There was no attempt to advance the scope through the aqueduct. For each of these video clips, the viewer had the ability to rewind and view each clip as many times as needed.

\section{Raters}

The "trained" group of raters included North American neurosurgeons with an interest in endoscopy who traveled to Uganda to learn the technique from Dr. Warf. These trained surgeons had on average performed $12 \mathrm{ETV} / \mathrm{CPC}$ procedures while in Uganda. Surgeons spent an average of 5 operative days in Uganda under direct supervision and instruction from Drs. Warf, John Mugamba, and/or Peter Ssenyonga. Upon return to their practices, each surgeon incorporated ETV/CPC into his/her routine procedures, which were performed an average of 1.5 times per month by each surgeon. Each surgeon had been performing ETV/ CPC for an average of 1.5 years. The "nontrained" group of raters was a group of neurosurgeons who had an interest in endoscopy but had not learned techniques directly from Dr. Warf. Among the nontrained group, only 1 surgeon works in a center where ETV/CPC is routinely performed. Each rater was blind to the findings of the other raters. There were 15 raters: 7 trained, 7 nontrained, and Dr. Warf.

Raters were asked to determine if cisternal scarring or aqueductal stenosis were present as defined by Warf et al. ${ }^{12}$ For the purposes of this study, a "scarred cistern contains anything more than typical strands of normal-appearing arachnoid. Once the Liliequist membrane is fenestrated, thickened membranes that even partially obstructed free passage of the endoscope into the cistern are considered to consist of scarring"12 (Fig. 1). To determine aqueduct
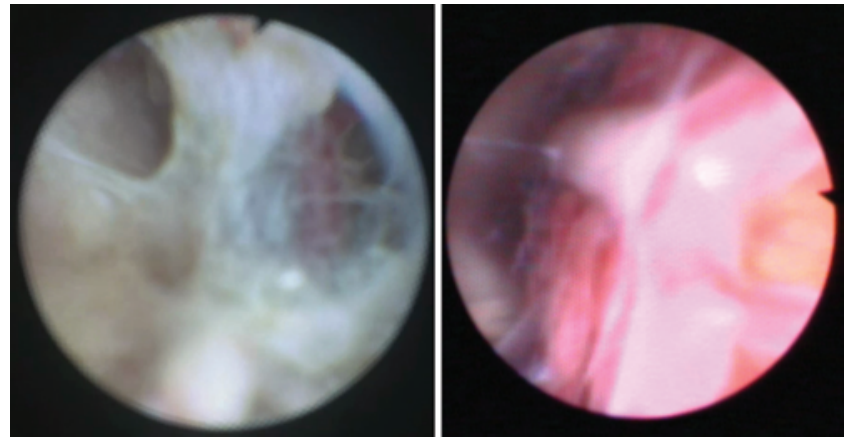

FIG. 1. Representative photos from the intraoperative ETV/CPC procedures. A scarred cistern (left) showing thickened membranes that obstruct the passage of the endoscope into the basilar cistern. An unscarred cistern status (right) is shown for comparison. Note the lack of adhesions or membranes obstructing the operator's view. Figure is available in color online only.

status, raters were provided with the following definition: "open is defined as clearly open or narrowed, closed is defined as clearly closed or a pinhole that is functionally obstructed" on direct inspection of the ostium without entering the aqueduct with the endoscope, which is consistent with the initial description and categorization of Warf et al. ${ }^{12}$ In this original description, the aqueduct was categorized as open, narrowed, or closed, but with the statistical analysis they were reclassified into 2 ratings: open or closed. In private correspondence, Dr. Warf explained that the narrowed aqueducts were considered closed if the aqueduct was "pinhole" or "functionally closed" (Fig. 2). Because of the need to use 2 ratings, this definition was used.

Each individual rater was sent an email link to surveys from Survey Monkey (www.surveymonkey.com; SurveyMonkey Inc.). None of the raters had access to any of the other raters' responses. To complete the survey, all raters had to respond to the entirety of the survey. The video clips were provided in a separate email link that was securely posted on Dropbox (Dropbox Inc.). For the presented video clips, all were recorded from ETV/CPC procedures that used equipment manufactured by the Karl Storz Co. and included a flexible endoscope (model 11282 BN; Karl Storz Co.). Video editing was performed using Final Cut Pro (Apple).

\section{Statistical Analysis}

The data analysis for this paper was generated using the Real Statistics Resource Pack software (Release 3.5; http://www.real-statistics.com/appendix/citation-real-sta tistics-software-website/). Interrater agreement between the 2 groups of observers was calculated using Fleiss' kappa coefficient $(\kappa) .^{2}$ Fleiss' kappa coefficient is related to Cohen's kappa, but is able to compare interrater reliability across more than 2 observers. ${ }^{1}$ A Fleiss' kappa coefficient less than 0 represents "poor agreement," $\kappa$ between 0.01 and 0.20 represents "slight agreement," $\kappa$ between 0.21 and 0.40 represents "fair agreement," $\kappa$ between 0.41 and 0.60 represents "moderate agreement," $\kappa$ between 0.61 and 0.80 represents "substantial agreement," and $\kappa$ between 0.81 and 1.00 represents "almost perfect agreement." As 


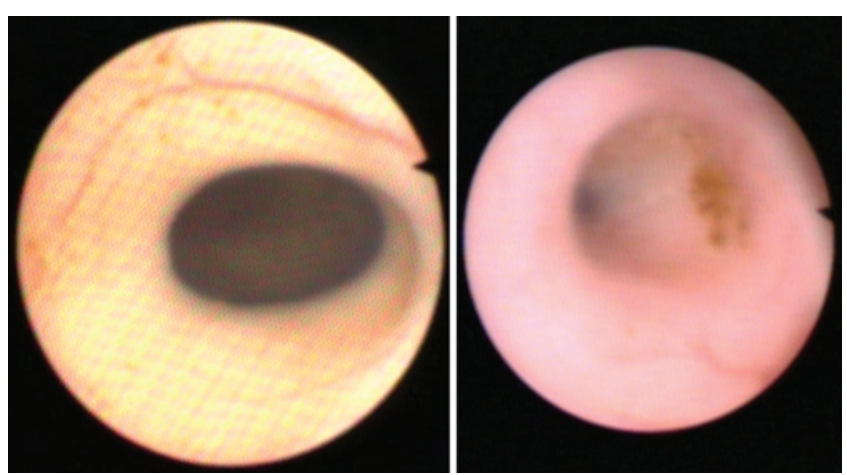

FIG. 2. Representative photos from the intraoperative ETV/CPC procedures assessing aqueduct patency. An open aqueduct (left) as seen by the surgeon compared with a closed aqueduct (right). Figure is available in color online only.

with Cohen's kappa, this scale is not universally accepted by all practitioners; however, it allows a general way to interpret values and understand the degree of agreement in our study. The scale and interpretation used here is commonly cited in other interrater reliability studies.

With Dr. Warf's assessment of cistern scarring and aqueduct patency as the reference answer, Fisher's 2-tailed exact test was used to assess if there was any statistically significant difference in the degree of disagreement be- tween the trained and nontrained groups for each video. Fisher's 2-tailed exact test was also used to assess any statistically significant difference in the degree of agreement between the trained and nontrained groups when all videos in the same category, as determined by Dr. Warf's answers, were taken as a group (scarred cisterns, unscarred cisterns, open aqueduct, and closed aqueduct).

\section{Results}

A total of 15 raters completed the survey, including Dr. Warf, 7 trained endoscopists, and 7 experienced but nontrained endoscopists (Fig. 3). For aqueduct status, among all raters, we had substantial agreement, with $\kappa$ of 0.663 (95\% CI 0.626-0.701). Within each group of endoscopists, there was substantial agreement, with $\kappa$ of 0.677 (95\% CI $0.593-0.761)$ for the trained group and $\kappa$ of 0.631 (95\% CI 0.547-0.715) for the nontrained group (Table 1). The identification of cistern scarring was less reliable. Among all raters, $\kappa$ of 0.536 (95\% CI $0.501-0.571)$ indicated moderate agreement. Within each group of endoscopists, there was moderate agreement, with $\kappa$ of $0.555(95 \% \mathrm{CI}$ $0.477-0.633)$ for the trained group and $0.542(95 \% \mathrm{CI}$ $0.464-0.620$ ) for the nontrained group (Table 1). Overall, there was substantial agreement in the status of aqueduct patency and moderate agreement in the degree of scarring of the cistern, regardless of the endoscopist's training.
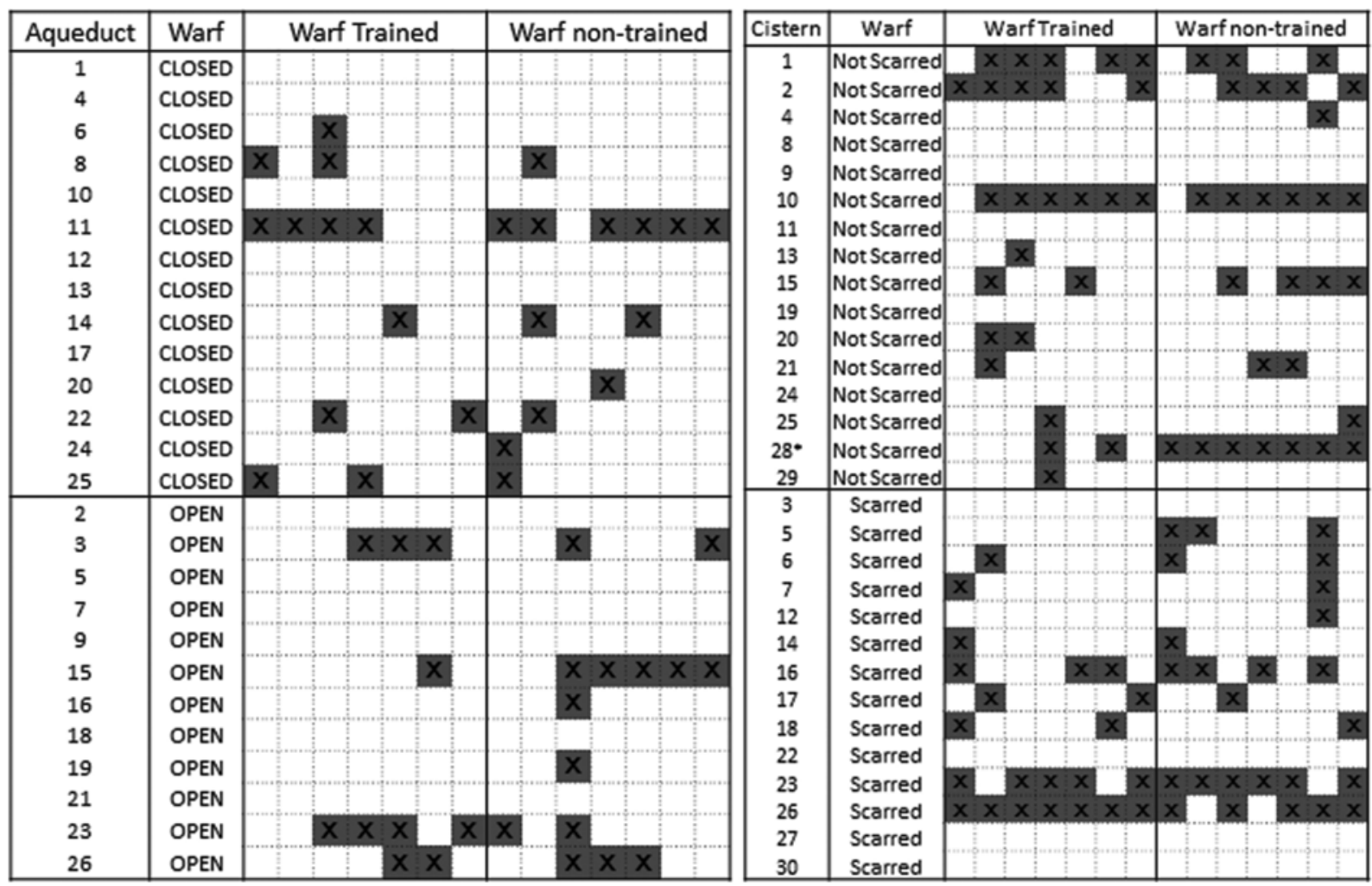

FIG. 3. Graphical representation showing aqueduct patency (left) and cisternal status (right) compared with Dr. Warf's reference answer. Note that $x$ indicates disagreement between the rater and Dr. Warf. Left: For aqueduct patency, there was no statistically significant difference between the amount of disagreement between the Warf-trained and nontrained groups. Right: For cisternal scarring, Video 28 was found to be statistically significant with regard to the amount of disagreement between the trained and nontrained groups. When comparing videos of the groups, as defined by Dr. Warf (e.g., closed aqueduct, scarred cistern), there was no statistically significant difference between the trained and nontrained groups. 
TABLE 1. Fleiss' kappa coefficients for all raters, trained raters, and nontrained raters for both aqueduct patency and cistern status*

\begin{tabular}{lcccccccc}
\hline & \multicolumn{3}{c}{ Aqueduct Patency } & & \multicolumn{3}{c}{ Cistern Status } \\
\cline { 2 - 3 } Value & All Raters & Trained & Nontrained & & All Raters & & Trained & Nontrained \\
\hline$\kappa$ & 0.663 & 0.677 & 0.631 & & 0.536 & 0.555 & 0.542 \\
\hline $95 \% \mathrm{Cl}$ & $0.626-0.701$ & $0.593-0.761$ & $0.547-0.715$ & & $0.501-0.571$ & $0.477-0.633$ & $0.464-0.620$ \\
\hline
\end{tabular}

* All $\kappa$ values were statistically significant $(p<0.05)$.

Using Dr. Warf's assessment of cistern scarring and aqueduct patency as the reference answer, only 1 cistern video (Video 28) showed a statistically significant difference in the degree of disagreement between the trained and nontrained groups by the Fisher's exact test ( $p=0.021)$. Comparing the videos grouped together by cistern or aqueduct status, as determined by Dr. Warf's reference answer, there was no statistically significant difference between the trained and nontrained groups $(\mathrm{p}>0.05)$ (Fig. 3).

\section{Discussion}

In this study, raters were given specific definitions for cistern scarring and aqueduct patency, and, regardless of training, the endoscopic surgeons reliably identified aqueduct patency with substantial agreement and cisternal scarring with moderate agreement based on Fleiss' kappa coefficient. ${ }^{5}$ This indicates that the definitions provided by Warf et al. ${ }^{11,12}$ are generalizable for endoscopic surgeons irrespective of additional training.

\section{Cisterns}

Cistern scarring has consistently been found to be a risk factor for ETV/CPC failure. ${ }^{6,711,12}$ This includes patient populations in both Africa (where infectious meningitis is the most common cause of scarring $)^{9}$ and North America (where intraventricular hemorrhage of prematurity is the most common cause of scarring). ${ }^{4}$ In both of these etiologies, Warf et al. identified scarring during the endoscopic inspection of the cisterns and found that it was correlated with failure. ${ }^{11,12}$ It is not clear if other surgeons would be able to reliably identify the same intraoperative findings.

It is important to ensure that surgeons can reliably identify scarring in order to guide intraoperative decision making about possible shunting and postoperative counseling of families. Additionally, the reliability of identifying scarred cisterns is important for studying ETV/CPC in clinical studies; otherwise the conclusions from studies will not be valid. As the technique becomes more widespread, surgeons who have not trained with Dr. Warf will be performing these procedures. It is important to know that the definitions can reliably guide operative decisions regarding scarring.

The ability of surgeons to identify scarred cisterns is moderately reliable, with a $\kappa$ of 0.536 (CI 0.501-0.571). While this is a relatively high level of reliability, there is potential room for improvement using other modalities to evaluate scarring. One disadvantage of intraoperative inspection is that surgery is required before the treatment decision can be made. Ideally, a patient's cistern status would be known before surgery, and the surgeon and family may alternatively choose to proceed with a shunt. Warf et al. reported in a small case series that on sagittal, balanced, steady-state, gradient-echo sequences (FIESTA, TrueFISP, CISS, bFFE) scarring could be identified and correlated with ETV/CPC failure. ${ }^{11}$ A potential direction of future research would be to determine if MRI could provide more reliable identification of cisternal scarring and preoperatively shape decision making.

Even if MRI is found to be superior to intraoperative inspection, most ETV/CPC procedures are currently performed in the developing world. Access to MRI is limited, and therefore intraoperative inspection of the cisterns will remain the only dependable method for the majority of cases performed, thereby making the findings from this study universally relevant.

\section{Aqueducts}

Closed aqueduct status correlates with ETV/CPC success in 1 study, ${ }^{12}$ but other studies have not had similar findings. ${ }^{7,8,10,11}$ This is surprising considering the high level of success that is correlated with ETV alone in patients with aqueductal stenosis and potentially indicates other important physiological changes that occur with the combination of ETV and CPC., ${ }^{3,13}$ While the identification of aqueductal stenosis can be obtained with cranial imaging, to date there are few studies that have looked at how intraoperative aqueduct ostium inspection compares with the findings on noninvasive imaging, especially in those aqueducts that are obstructed and functionally closed versus clearly open. ${ }^{9}$ Our study indicates that regardless of endoscopic training, the application of Dr. Warf's definition for aqueduct patency can be applied with substantial agreement between raters consistently in the intraoperative setting. This high level of reliability allows for endoscopists in settings without readily available imaging to make intraoperative assessments that may affect their decision making.

\section{Limitations}

An assumption of this study is that an edited, shortened video is a valid representation or reenactment of the endoscopy experience, thereby allowing the rater to analyze and rate the status of the cisterns and aqueduct as they would if they were performing the endoscopy themselves. Certainly, this representation should be more valid than still pictures; however, it is possible that unedited video (while exhaustingly long to watch and rate) may be more valid. Also, it is possibly only valid when the rating endoscopic surgeon is controlling the scope and making observations 
while navigating the scope. Of note, as demonstrated in Fig. 3, both groups often differed from Dr. Warf's ratings. Although the raters were relatively reliable in rating the cisterns and aqueducts when compared with Dr. Warf as the gold standard (Fig. 3), both groups of raters often differed from Dr. Warf. This could represent us having a reliable rating system, but it questions the validity. Because the outcomes of the ETV/CPC procedures could not be linked to the videos, the validity could not be confirmed. It is certainly possible that Dr. Warf's ratings of the videos were a representation of his experience more than a strict adherence to the definitions.

\section{Conclusions}

The results from this reliability study indicate that among multiple observers there is good agreement between Warf-trained and nontrained observers about the degree of cistern scarring and aqueduct patency. The trained versus nontrained groups had comparable agreement in terms of the degree of cisternal scarring and aqueductal patency. The high Fleiss' kappa coefficient indicates that Dr. Warf's definitions of cisternal scarring and aqueduct patency are generalizable.

\section{References}

1. Cohen J: Weighted kappa: nominal scale agreement with provision for scaled disagreement or partial credit. Psychol Bull 70:213-220, 1968

2. Fleiss JL: Measuring nominal scale agreement among many raters. Psychol Bull 76:378-382, 1971

3. Kulkarni AV, Drake JM, Mallucci CL, Sgouros S, Roth J, Constantini S: Endoscopic third ventriculostomy in the treatment of childhood hydrocephalus. J Pediatr 155:254-259, 259.e1, 2009

4. Kulkarni AV, Riva-Cambrin J, Butler J, Browd SR, Drake JM, Holubkov R, et al: Outcomes of CSF shunting in children: comparison of Hydrocephalus Clinical Research Network cohort with historical controls: clinical article. J Neurosurg Pediatr 12:334-338, 2013

5. Landis JR, Koch GG: The measurement of observer agreement for categorical data. Biometrics 33:159-174, 1977

6. Marano PJ, Stone SSD, Mugamba J, Ssenyonga P, Warf EB, Warf BC: Reopening of an obstructed third ventriculostomy: long-term success and factors affecting outcome in 215 infants. J Neurosurg Pediatr 15:399-405, 2015

7. Stone SSD, Warf BC: Combined endoscopic third ventriculostomy and choroid plexus cauterization as primary treat- ment for infant hydrocephalus: a prospective North American series. J Neurosurg Pediatr 14:439-446, 2014

8. Warf BC: Comparison of endoscopic third ventriculostomy alone and combined with choroid plexus cauterization in infants younger than 1 year of age: a prospective study in 550 African children. J Neurosurg 103 (6 Suppl):475-481, 2005

9. Warf BC: Hydrocephalus in Uganda: the predominance of infectious origin and primary management with endoscopic third ventriculostomy. J Neurosurg 102 (1 Suppl):1-15, 2005

10. Warf BC, Campbell JW: Combined endoscopic third ventriculostomy and choroid plexus cauterization as primary treatment of hydrocephalus for infants with myelomeningocele: long-term results of a prospective intent-to-treat study in 115 East African infants. J Neurosurg Pediatr 2:310-316, 2008

11. Warf BC, Campbell JW, Riddle E: Initial experience with combined endoscopic third ventriculostomy and choroid plexus cauterization for post-hemorrhagic hydrocephalus of prematurity: the importance of prepontine cistern status and the predictive value of FIESTA MRI imaging. Childs Nerv Syst 27:1063-1071, 2011

12. Warf BC, Kulkarni AV: Intraoperative assessment of cerebral aqueduct patency and cisternal scarring: impact on success of endoscopic third ventriculostomy in 403 African children. J Neurosurg Pediatr 5:204-209, 2010

13. Warf BC, Tracy S, Mugamba J: Long-term outcome for endoscopic third ventriculostomy alone or in combination with choroid plexus cauterization for congenital aqueductal stenosis in African infants. J Neurosurg Pediatr 10:108-111, 2012

\section{Disclosures}

The authors report no conflict of interest concerning the materials or methods used in this study or the findings specified in this paper.

\section{Author Contributions}

Conception and design: Rocque, Riva-Cambrin, Naftel. Analysis and interpretation of data: He. Drafting the article: He, Naftel. Critically revising the article: all authors. Reviewed submitted version of manuscript: all authors. Approved the final version of the manuscript on behalf of all authors: He. Statistical analysis: He, Shannon, Naftel. Administrative/technical/material support: Gannon. Study supervision: Naftel.

\section{Correspondence}

Lucy He, Pediatric Neurosurgery, Vanderbilt University Medical Center, 9222 Doctors' Office Tower, 2200 Children's Way, Nashville, TN 37232-9557.email: lucy.he@vanderbilt.edu. 